\title{
Magnetic field sector structure and origins of solar wind streams in 2012
}

\author{
Yulia Shugay ${ }^{1, *}$, Vladimir Slemzin ${ }^{2}$, and Igor Veselovsky ${ }^{1,3}$ \\ 1 Skobeltsyn Institute of Nuclear Physics, Lomonosov Moscow State University, Moscow 119991, Russia \\ ${ }^{*}$ Corresponding author: j.shugai@srd.sinp.msu.ru \\ 2 Lebedev Physical Institute, Russian Academy of Sciences, Moscow 119991, Russia \\ 3 Space Research Institute (IKI), Russian Academy of Sciences, Moscow 117997, Russia
}

Received 4 December 2013 / Accepted 26 June 2014

\begin{abstract}
The origins of the solar wind and the interplanetary magnetic field sector structure in the beginning of the magnetic polarity reversal of 24th solar cycle were investigated using the Wilcox Solar Observatory magnetic field measurements and their products as well as the solar wind data from ACE and the SDO/AIA EUV images. The dominance of the quadrupole harmonics in the solar magnetic field in this period resulted in a four-sector structure of the interplanetary magnetic field. The dominating source of recurrent high-speed solar wind stream was a large trans-equatorial coronal hole of negative polarity evolving in the course of the polarity reversal process. The contribution of ICMEs to the high-speed solar wind did not exceed $17 \%$ of the total flux. The solar wind density flux averaged over the year amounted to $1 \times 10^{8} \mathrm{~cm}^{-2} \mathrm{~s}^{-1}$ which is considerably lower than the typical long-term value $\left(2-4 \times 10^{8} \mathrm{~cm}^{-2} \mathrm{~s}^{-1}\right)$. The slow-speed component of solar wind density flux constituted in average more than $68 \%$ of the total flux, the high-speed component was about $10 \%$, reaching the maximum of $32 \%$ in some Carrington rotations.
\end{abstract}

Key words. solar cycle - coronal magnetic field - sources of solar wind - coronal hole - interplanetary magnetic field - solar wind

\section{Introduction}

The solar magnetic field structure and its variations are the main factors governing the conditions of space weather and, finally in long-term scale, space climate in the heliosphere. The solar magnetic field varies within the Hale cycles lasting about 22 years. The global magnetic field on the Sun is formed by the superposition of the dipole, quadrupole, and octupole harmonics. The polarity reversal process in the heliosphere can be represented as the turn and flip of the interplanetary current sheet. During the vertical position of the dipole axis observed at solar minimum, the current sheet is almost flat and lies near the solar equator. However, it gets tilted and warped, when quadrupole and higher moments are starting to dominate. The position of the current sheets determines the number of magnetic sectors of the interplanetary magnetic field observed at Earth's orbit. The dominance of the quadrupole harmonics in the solar magnetic field leads to the four-sector structure of the interplanetary magnetic field (IMF) in the plane of the ecliptic, and even at sufficiently high latitudes. It should be noted that the number of high-speed solar wind (SW) streams does not necessarily correspond to the number of sectors (Veselovsky et al. 2006; Wang et al. 2014), but it depends on the existence and the location of large coronal holes (CHs) on the Sun and on sporadic solar activity.

The polarity reversal process accompanied by shifts of the polar $\mathrm{CHs}$ to the equator permits to disclose the direct association between $\mathrm{CHs}$ as the sources of high-speed recurrent $\mathrm{SW}$ streams and parameters of SW measured "in situ". Several studies have investigated how the long-term $\mathrm{CHs}$ evolution is related to the changes in the global solar magnetic field over solar cycle (e.g. Bilenko 2002; Sanderson et al. 2003; Wang 2009; Obridko \& Shelting 2011). CHs originate in sufficiently large unipolar regions with predominantly open configuration of the magnetic field lines. Around solar minimum, when the solar field is dipolar and the position of the magnetic field dipole axis is close to vertical, the $\mathrm{CHs}$ are located at the poles. When the dipole axis begins to incline, the polar $\mathrm{CHs}$ decrease in size and fully disappear at the inclination angle of nearly $90^{\circ}$. The parts of the polar CHs extended to low latitudes break off into groups of small $\mathrm{CHs}$ when the magnetic pole moves to lower latitudes. When the magnetic quadrupole moment dominates over dipole one, a number of $\mathrm{CHs}$ of the same polarity appear at mid-latitudes.

The Wilcox Solar Observatory (WSO) source surface synoptic charts (http://wso.stanford.edu/synsourcel.htm) clearly show the polarity reversal process beginning in 2012 near the maximum of the current 24 solar cycle. Figure 1 (top panel) shows the calculated tilt of the heliospheric current sheet against the rotation axis of the Sun (http://wso.stanford.edu/gifs/ Tilts.gif). The global coronal magnetic field in the Southern hemisphere was positive at the rising phase before the solar maximum, and it is expected to be negative here at the declining phase. The quadrupole harmonics dominate in the solar magnetic field and reach the maximum strength in mid-2012 when the decrease of the dipole component was observed (Fig. 1, bottom panel). The comparison with the preceding cycles shows that such evolution of the global magnetic field in the current cycle is rather typical, except for deeper decrease of the dipole component in the solar minimum. 

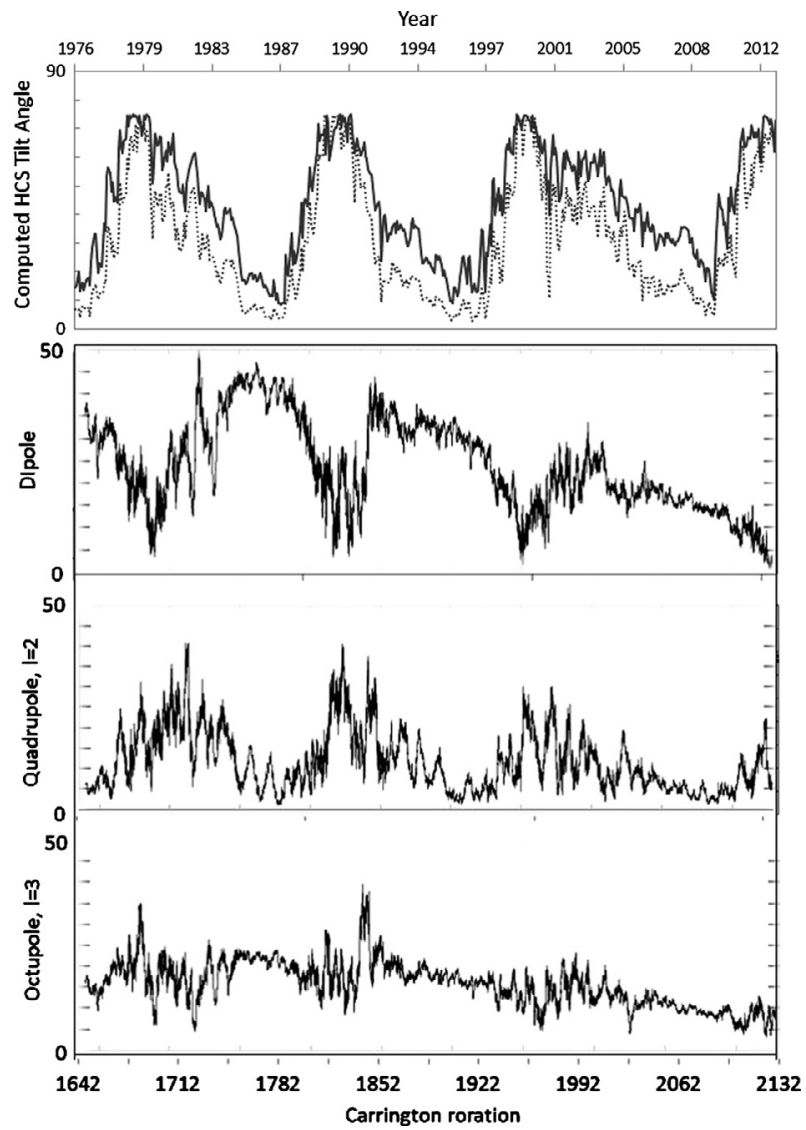

Figure 1. Top panel: computed maximum inclination of the heliospheric current sheet illustrating the flip of the global magnetic field for 1976-2012 (Carrington rotations 1642-2132). The solid line is the simulation with the classic PFSS model using the line-ofsight boundary condition in the photosphere including significant polar field correction; the dashed line is the model with higher source surface radius $R_{\mathrm{S}}=3.25$, it uses a radial boundary condition at the photosphere without polar field correction. Bottom panel: the total strength of the first three multipole components (dipole, quadrupole, and octupole, from top to bottom respectively) of the solar magnetic field during Carrington rotations 1642-2132. The data was provided by the Wilcox Solar Observatory (http://wso.stanford.edu)

The formation and evolution of $\mathrm{CHs}$ were considered by many researches, being most discussed in detail by Wang \& Sheeley (2004), Wang (2009, 2010a) and Wang et al. (2010b). The CHs form above unipolar regions, which originate from the decay of active regions (AR) and evolve through the flux transport processes: supergranular diffusion, differential rotation, and meridional flow. The interchange reconnections between $\mathrm{CH}$ and nearby ARs influence the formation of the CH boundaries (Wang \& Sheeley 2004).

The properties of the SW streams in different phases of the solar cycle are closely related to the configuration of the global magnetic field and its origins in the corona. Coronal mass ejections and large-scale $\mathrm{CHs}$ are known to be main sources of fast SW streams. Identification of sources of the slow SW streams so far remains to be not so evident. Based on the empirical relation between SW speed and expansion factor, Wang (2009) concluded that most of the slow SW comes from the boundaries of $\mathrm{CHs}$ as well as from small coronal holes that form at the edges of ARs. The relationship between large-scale magnetic fields, CHs and ARs was studied by Mogilevsky et al. (1997). The researchers found out that boundaries of CHs are affected by disturbances that occur in ARs or active complexes in the process of their evolution.

Among the non-coronal hole sources of slow SW streams, there are streamers (Sheeley et al. 1997), quasi-stationary outflows from ARs (Kojima et al. 1999; Liewer et al. 2001; Neugebauer et al. 2002), particularly, from those adjacent to small low-latitude CHs (Liewer et al. 2004; Harra et al. 2008; Del Zanna 2008; Slemzin et al. 2013), regions of magnetic polarity inversions on tops of closed loops (Owens et al. 2013).

In this work we are focusing on the structure and evolution of global coronal magnetic field, long-living $\mathrm{CHs}$, sector structure of IMF and parameters of the SW streams near maximum of solar cycle 24 based on magnetic field extrapolations as well as on the EUV images. We consider the evolution of fast-speed and slow-speed components of SW during the year and their relationship with the parameters of $\mathrm{CHs}$ and the solar activity.

\section{Data and analysis}

Configuration of the global magnetic field in the corona was analyzed using the magnetic field maps from WSO. Those maps are calculated from the data of photospheric field observations using the potential field model (Hoeksema et al. 1982). The details of the computation can be found elsewhere (http://wso.stanford.edu/synsourcel.html). The images of the Sun were obtained by Solar Dynamics Observatory/ Atmospheric Imaging Assembly (SDO/AIA; Hurlburt et al. 2012). The hourly values of the IMF and the SW parameters were measured at the ACE (Advanced Composition Explorer) spacecraft by means of the instruments Magnetic Field Experiment (MAG; Smith et al. 1998) and Solar Wind Electron, Proton, and Alpha Monitor (SWEPAM; McComas et al. 1998).

It is well known that good estimates of recurrent high-speed SW could be obtained from the models that use the relationship between SW parameters and the position and size of CHs determined by the EUV imaging (Vrsnak et al. 2007; Krista \& Gallagher 2009; Obridko et al. 2009). Therefore, to identify $\mathrm{CHs}$ and their associated SW streams, we used solar images obtained by SDO/AIA in the $193 \AA$ spectral channel and the $\mathrm{CH}$ areas calculated using the algorithm described by Shugay et al. (2011). The data from SDO/AIA was taken in the standard FITS-format, the sampling rate being one image per hour. In the images, only those regions, which covered more than $0.4 \%$ of the total solar disk area and had the intensity below the threshold value, were treated as CHs. The threshold intensity was calculated as $I_{\text {mean }} k$, where $I_{\text {mean }}$ is the average intensity of the solar disk without taking into account the limb brightening, and $k$ is the empirical threshold coefficient, taken equal to 0.3 for the SDO/AIA images centered at $193 \AA$. Hereafter we are using the relative areas of $\mathrm{CHs}$ defined as the ratio of the geometrical $\mathrm{CH}$ areas to the area of the solar disk. The SW sources positioned in the central part of the solar disk produce measurable contribution to SW near the Earth. Therefore, we calculated the area of $\mathrm{CHs}$ within the region confined between $\pm 30^{\circ}$ longitudes from the central meridian of the Sun and between $\pm 50^{\circ}$ latitudes from the solar equator. Figure 2 shows the values of the $\mathrm{CH}$ areas for the period January-November 2012 as white curves below the coronal field synoptic maps. Figure 3 shows the synoptic maps of solar corona constructed from the SDO/AIA $193 \AA$ images with the $\mathrm{CH}$ boundaries contoured. 

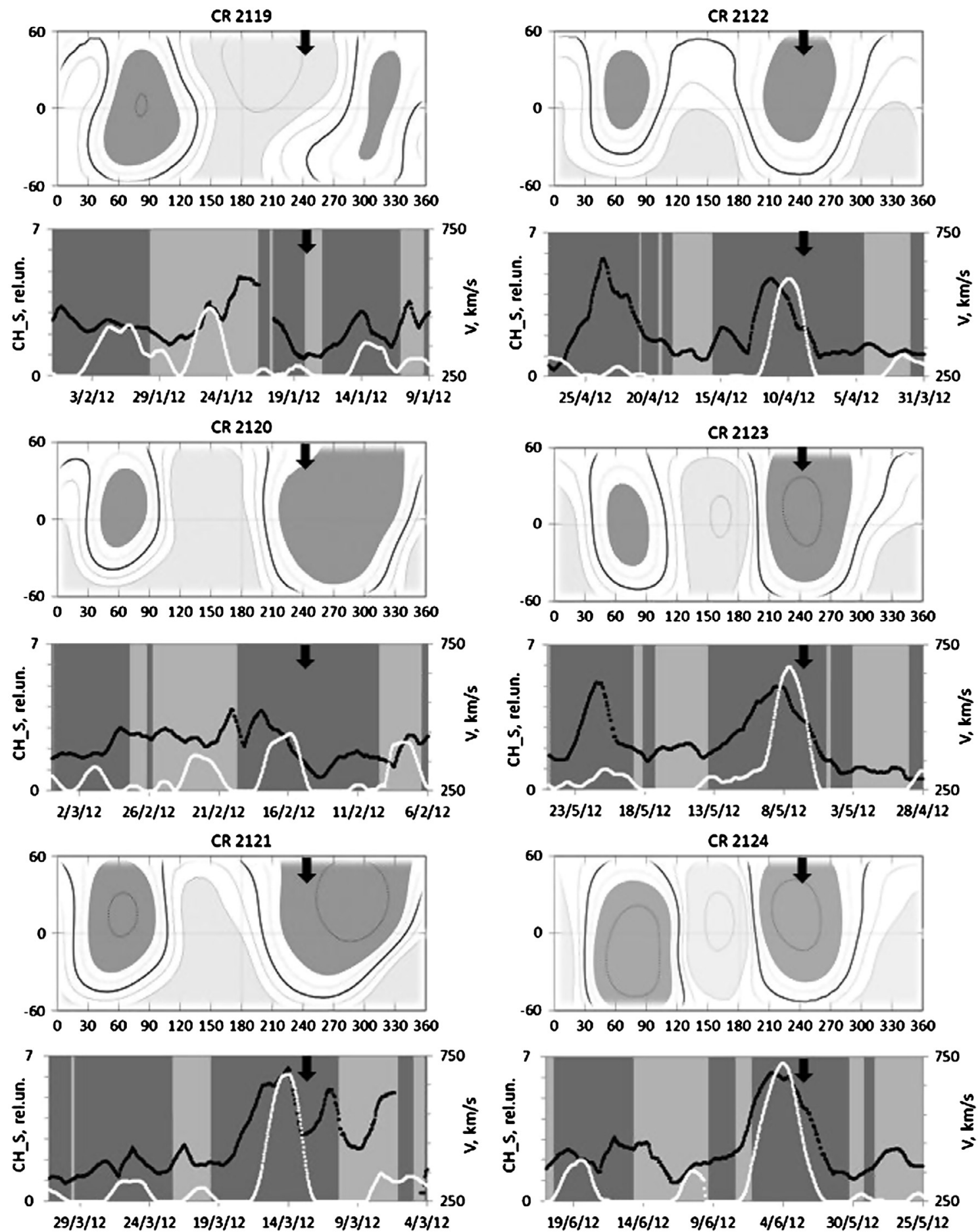

Figure 2a. Evolution of the coronal magnetic field, the areas of CHs and the SW velocity and IMF polarity during CR 2119-2124 recalculated to the source surface. Top panel for each CR: coronal magnetic field maps at the source surface from WSO (positive polarity field are light gray, negative are dark gray). Bottom panel for each CR: IMF polarity (dark gray areas represent positive polarity) as the sign of the $24 \mathrm{~h} \mathrm{moving}$ average of $B x$-component of IMF in the GSM coordinate system, and SW velocity (black curve). White curve is the area of the CHs calculated using the SDO/AIA data. The time axis is directed from right to left for easier comparison with the coronal field map. Black arrows show the analyzed region of the coronal magnetic field associated with the LCH (see Sect. 3).

To analyze correlation with the coronal magnetic field maps, the SW data was recalculated to the source surface (SS) as a function of heliographic longitude using the ballistic model. MacNeice et al. (2011) have validated "community models" of the SW propagation in the heliosphere, such as WSA/ENLIL, and found that, due to uncertainty of the initial static photospheric magnetograms, no sophisticated heliospheric model does provide better accuracy in comparison with the simple ballistic model based on the Archimedean spiral approximation (Nolte \& Roelof 1973). In the ballistic model the longitude at SS corresponding to the ACE data is calculated as:

$$
L_{\mathrm{SS}}(t)=L_{\mathrm{E}}(t)-\omega \times D(t) / V_{\mathrm{E}}(t),
$$



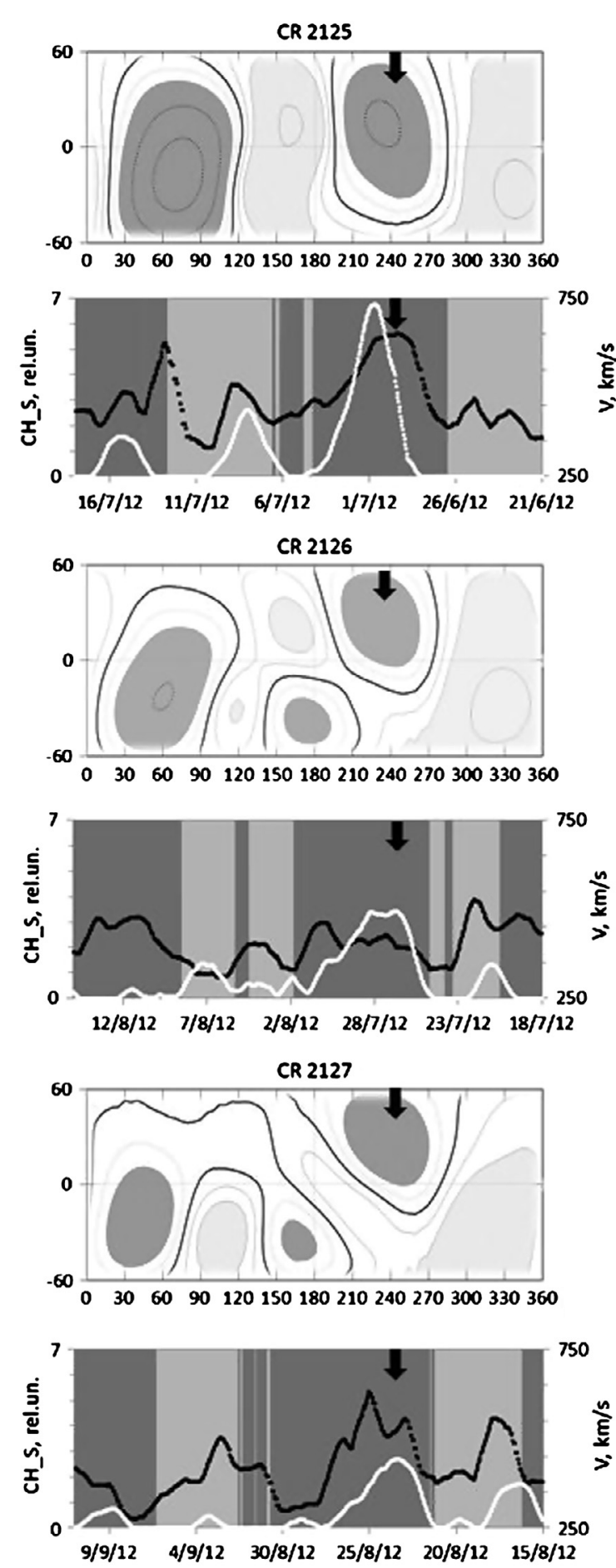

Figure 2b. Same as Figure 2a, but during CR 2125-2130.

where $L_{\mathrm{E}}(t)$ is the solar longitude as seen from Earth at the time $t, \omega$ is the synodical speed of the solar rotation near the equator $(13.23 \%$ day), $D(t)$ is the distance between the Sun and the spacecraft, $V_{\mathrm{E}}$ is the solar wind speed near the Earth's orbit. The typical uncertainty of this approach is about $\pm 10^{\circ}$. Polarity of IMF (dark gray and light gray areas in Figs. $2 \mathrm{a}$ and $2 \mathrm{~b}$ below the magnetic field maps) is calculated as the sign of $24 \mathrm{~h}$ moving average of $B x$-component of IMF in the GSM coordinate system.

It is worth noting that here we use the ballistic model only for bounding the temporal record of the ACE data to longitudes
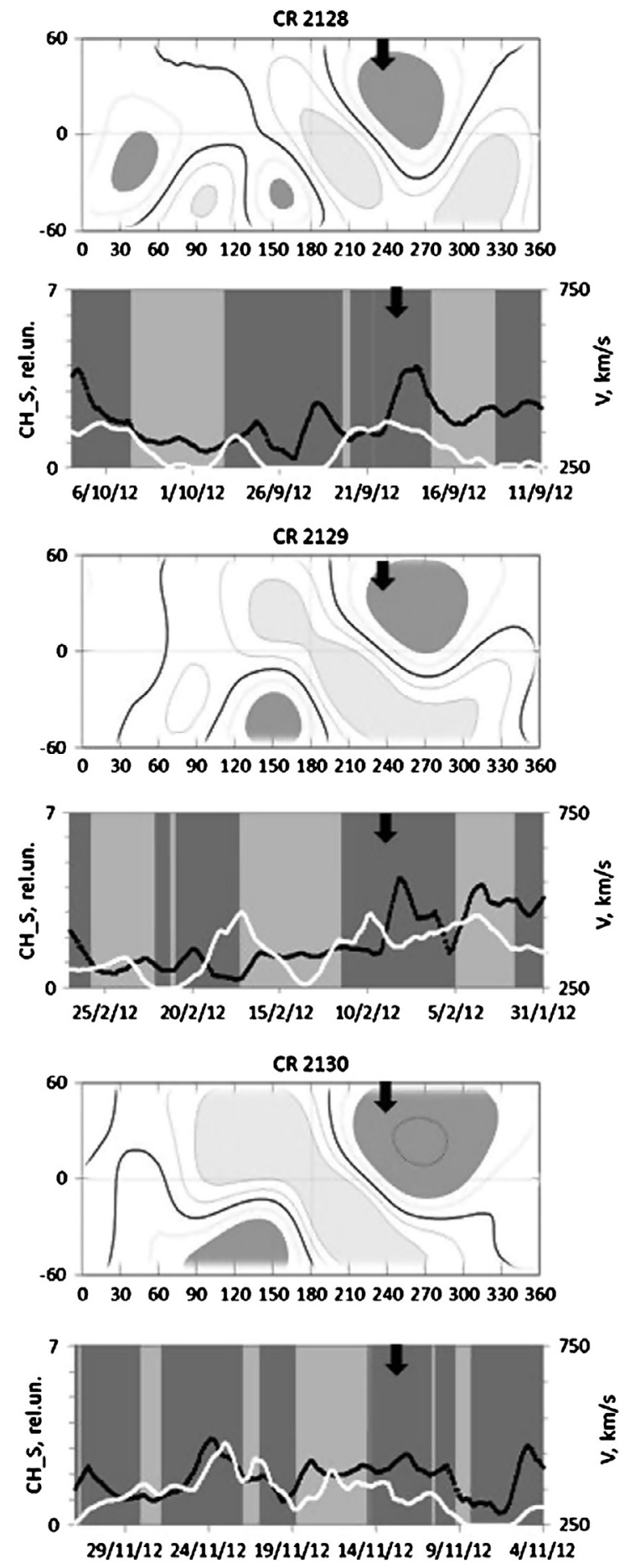

at SS but not for evaluation of the SW parameters. Whereas the SW velocity can be taken as constant on the way from SS to the Earth, the magnetic field strength and SW density vary according to the field expansion factor, which is in turn defined by the magnetic field in the coronal base (Wang 2010a).

Figures $2 \mathrm{a}$ and $2 \mathrm{~b}$ display the maps of the coronal magnetic field from the WSO web site during Carrington rotations (CR) 2119-2130 (January-November 2012), which are compared with the areas of CHs obtained by SDO/AIA at $193 \AA$, the sector structure of IMF and the SW streams velocity recalculated to the source surface. Keep in mind that negative radial 

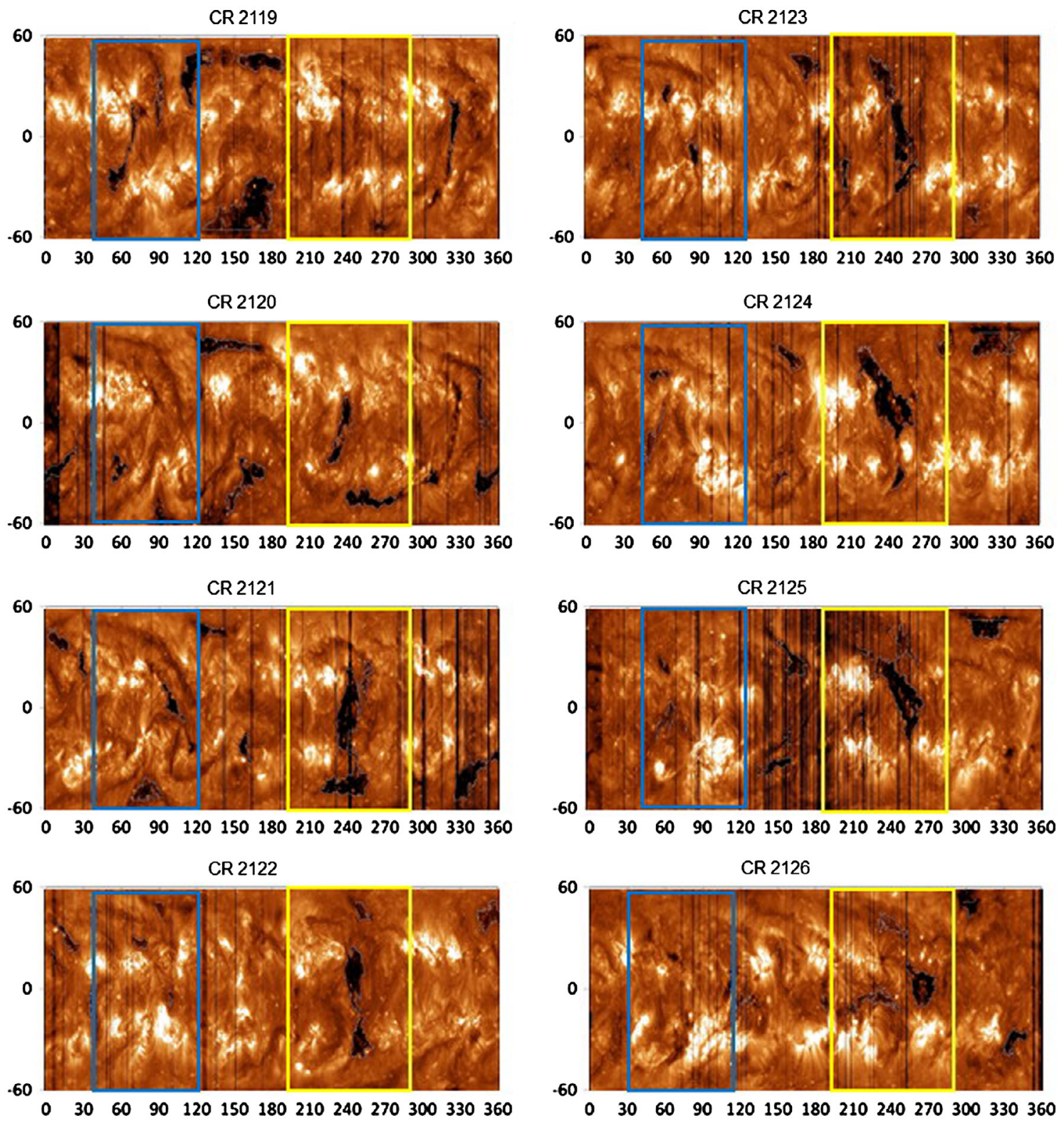

Figure 3. Synoptic maps constructed from the EUV images in the channels $193 \AA$ obtained by SDO/AIA during the period from January to August, 2012 (CR 2119-2126). The LCH located in one of the negative polarity regions of the coronal magnetic field is shown inside the yellow frame. Small CHs located in other negative polarity region are shown inside the blue frame.

component of the magnetic field pointed into the Sun corresponds to positive radial component in the GSM coordinate system.

\section{Magnetic field structure and origins of solar wind in 2012}

The solar magnetic field reversal during cycle 24 started in the northern hemisphere (Svalgaard \& Kamide 2013). The northern polar $\mathrm{CH}$ disappeared in 2011, but the southern $\mathrm{CH}$ still existed at the beginning of 2012 (Fig. 3). At that time, the dipole axis was near the equatorial plane, as we can see at the top panel of Figure 1. The superposition of dipolar, quadrupolar, and octupolar harmonics of magnetic field (Fig. 1, bottom panel) led to asymmetry in longitude and latitude of the hemispheric current sheet and to the formation of four global patterns of the coronal magnetic field of positive and negative polarity at sufficiently high latitudes in 2012 (Figs. 2a and 2b, coronal maps). Wang et al. (2014) described the synoptic map of the source-surface field during CR 2123 and 2124 as "two roughly circular neutral lines, centered near the equator and separated by $\sim 180^{\circ}$ in longitude. Each of the two circular neutral lines at the source surface is centered above negative-polarity coronal hole". The CHs located inside the negative polarity regions can be seen in Figure 3 (within the yellow and blue frames).

An isolated transequatorial large $\mathrm{CH}(\mathrm{LCH})$ of the negative polarity was observed during CR $2120-2126$ in the equatorial region (Fig. 3, within the yellow frame). That $\mathrm{LCH}$ was the main source of the recurrent high-speed SW streams in the Earth's orbit from February to September, 2012 (Figs. 2a and $2 \mathrm{~b}$, marked arrows). Another $\mathrm{CH}$ of the negative polarity 


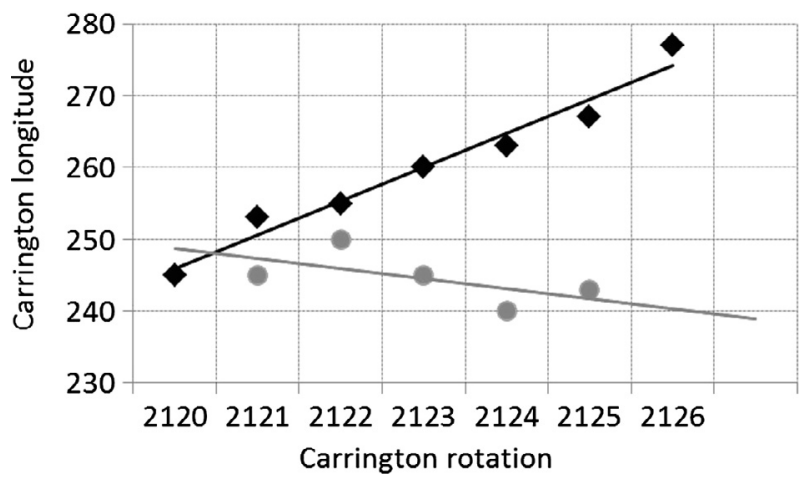

Figure 4. Longitudinal shifts of the LCH front boundary at the equator (black diamonds) and at the latitude of $30^{\circ} \mathrm{N}$ (gray circles) during CR 2120-2126. The lines represent the linear approximations.

had a small size, so it's contour is not discernible in the EUV images in Figure 3, within the blue frame. During CR 2122 and 2123 some of high-speed streams were connected with these $\mathrm{CHs}$.

Transient flows contribute to high-speed SW streams, as well. For example, the SW streams $\left(700-750 \mathrm{~km} \mathrm{~s}^{-1}\right)$ observed during CR 2121 were produced by coronal mass ejections (CME) associated with the flares of classes $\mathrm{M}$ and $\mathrm{X}$ that happened on March 7-9 in the AR located in front of the LCH (Figs. 2a, 2b, and 3). The high-speed stream observed during CR 2125 (11-15 July) on the border between positive and negative polarity IMF sectors was also caused by the CME which initiated the interplanetary coronal mass ejection (ICME). The small CHs near ARs are observed in the positive polarity regions at different Carrington latitudes. Such $\mathrm{CHs}$ could be the sources of SW flows with the speed of less than $500 \mathrm{~km} \mathrm{~s}^{-1}$.

The $\mathrm{LCH}$ that appeared in the negative polarity region of the coronal magnetic field in CR 2120 was observed during CR 2120-2126 (Figs. 2a, 2b, and 3). During that time, the quadrupole component increased and reached its peak in mid2012 , its total strength exceeding the strength of the dipole component (Wang et al. 2014). During CR 2126-2130 the stable quadrupolar structure significantly and rapidly changed. The southern part of the negative polarity regions broke away and moved eastwards, and then merged with the second negative region that existed at longitudes $\sim 30-120^{\circ}$ during CR 2119-2126. The strength of the quadrupole component decreased after mid-2012 (Fig. 1, bottom panel). During CR 2126, the large $\mathrm{CH}$ considerably reduced in size so only its equatorial part at latitudes $5^{\circ} \mathrm{S}$ to $15^{\circ} \mathrm{N}$ remained (Fig. 3, inside the yellow frame). During CR 2127-2128, this remnant of the $\mathrm{CH}$ significantly decreased and fully disappeared during CR 2129. Figures $2 \mathrm{a}$ and $2 \mathrm{~b}$ show the considerable difference between the configurations of the coronal magnetic field during CR 2120 (when the LCH appeared) and CR 2129 (when the $\mathrm{LCH}$ disappeared). Thus, disappearance of the $\mathrm{LCH}$ can be related to the strength of the quadrupolar harmonics of the magnetic field.

During CR 2120-2126, the LCH boundaries moved in longitude due to the $\mathrm{CH}$ differential rotation (Fig. 4). We have estimated the longitudinal shift of the front LCH boundary at two different latitudes $\left(0^{\circ}\right.$ and $\left.30^{\circ} \mathrm{N}\right)$. The $\mathrm{CH}$ boundary near the solar equator shifted approximately by $5^{\circ}$ per rotation in the direction of rotation, while the LCH front boundary near $30^{\circ} \mathrm{N}$ latitude had very small shift, only $\sim 2^{\circ}$ over all five rotations in the opposite direction. The equatorial parts of the $\mathrm{LCH}$ rotate slightly faster than the mid-latitude parts of the $\mathrm{LCH}$ with the period shorter than the Carrington rotation period (27.3 days).

The $\mathrm{CHs}$ rotation during the maximum of the solar cycle 21 (CR 1716-1739) was analyzed in the work by Shelke \& Pande (1985). The authors concluded that the CHs observed in helium $10830 \AA$ exhibit differential rotation. They found that $\mathrm{CHs}$ located at higher latitudes in northern and southern hemispheres $\left(20-40^{\circ}\right.$ and $\left.40-60^{\circ}\right)$ moved in opposite directions, if compared with equatorial $\mathrm{CHs}$. The longitudinal shifts were about $10^{\circ}$ and $20^{\circ}$ per rotation for latitude zones of $20-40^{\circ}$ and $40-60^{\circ}$, respectively.

In our case, the equatorial part of the $\mathrm{LCH}$ similarly rotates faster than its mid-latitude part. However, the difference in the inclination angle between its parts at the equator and at the $30^{\circ} \mathrm{N}$ latitude is only $\sim 5^{\circ}$ per rotation. Due to differential rotation of photospheric field, a rotation period of the equatorial part of the $\mathrm{LCH}$ is close to 26.9 days as compared to that of the mid-latitude part of the $\mathrm{LCH}$, which is near 27.3 days. The similar period of 26.9 days was found by Wang et al. (2010b) for the ARs located near equator out of which the CHs were formed.

\section{Structure and variation of the solar wind density flux in 2012}

As we can see in Figures $2 \mathrm{a}$ and $2 \mathrm{~b}$, bottom panels, during CR 2019-2125, the four-sector structure of IMF was associated with one, two or three high-speed SW streams (velocity is more than $450 \mathrm{~km} \mathrm{~s}^{-1}$ ). During CR 2120-2125, the recurrent SW streams with the speed exceeding $600 \mathrm{~km} \mathrm{~s}^{-1}$ were observed with mainly positive polarity of IMF (Fig. 5, left). These high-speed SW streams are associated with the recurrent LCH. The strong asymmetry of the magnetic field-velocity distribution for the high-speed SW flows was observed only during CR 2119-2125 when the LCH existed. The bulk of SW flows had the SW velocity of nearly $400 \mathrm{~km} \mathrm{~s}^{-1}$. The fieldvelocity distribution for the slow-speed SW flows (less $450 \mathrm{~km} \mathrm{~s}^{-1}$ ) displays only the small asymmetry for the $B x$ component of positive polarity.

During CR 2126, when the LCH area decreased, the SW velocity dropped below $600 \mathrm{~km} \mathrm{~s}^{-1}$. Only during CR 2127, although the LCH area has not changed (Fig. 2b, white curve), the speed of SW flows still increased up to $600 \mathrm{~km} \mathrm{~s}^{-1}$. The distribution of the SW velocity versus $B x$-component of IMF was more symmetrical during CR 2126-2131 than during CR 2119-2125 (Fig. 5, right). The bulk of the SW flow has the velocity near $350 \mathrm{~km} \mathrm{~s}^{-1}$ during CR 2126-2131. Striking difference in the distribution of IMF polarity versus SW velocity is observed before and after reconfiguration of the quadrupolar structure of the coronal magnetic field that has started during CR 2126.

To evaluate relative contributions of the high-speed and slow-speed SW components to the total density flux balance and to estimate the relationship between those and the largescale magnetic field, we analyzed the variation of the SW density flux $n V$, where $n$ and $V$ are the density and velocity of protons taken from the ACE hourly data. The distributions of the average SW density flux versus velocity, separately for the positive and negative $B x$-components of the IMF, are 

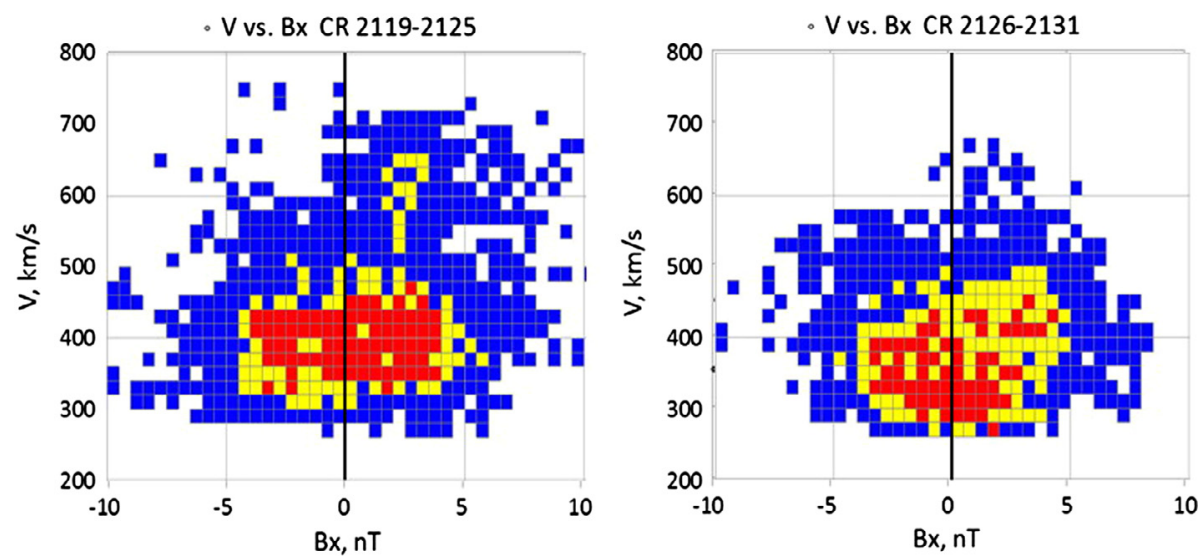

Figure 5. Scatter plot of hourly values of the SW velocity versus the $B x$-component of IMF during CR 2119-2125 (left) and CR $2126-2131$ (right). The blue bars correspond to the counts less than 10, the yellow bars - from 10 to 20 and the red bars - more than 20 .
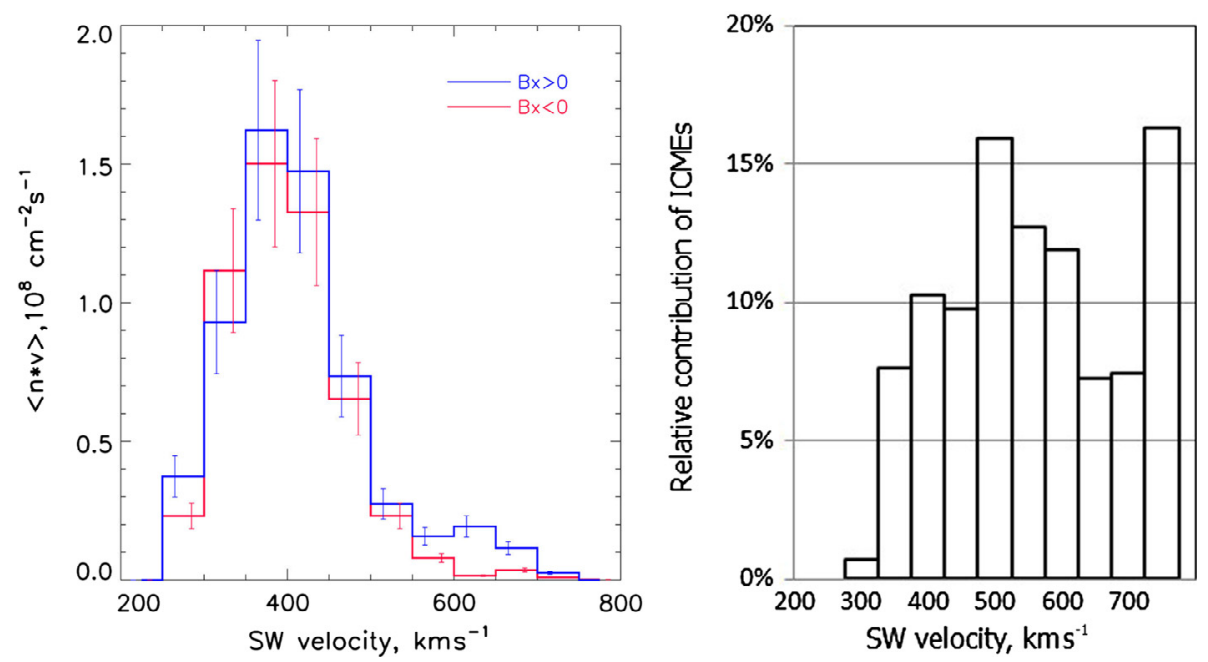

Figure 6. Left: variation of the mean SW density flux averaged over 2012 versus the SW velocity. The SW density flux with the positive polarity $B x$-component of the IMF is marked by the blue line, and that with negative polarity is marked by the red line. Right: relative contribution of ICME (in \%) to the SW flux in 2012 as function of SW speed.

presented in Figure 6, left. The error bars in these histograms correspond to the $20 \%$ uncertainty in the ACE density values estimated as the worst case from the divergence of the ACE and Wind SW data at the OMNIWEB site (http://omniweb. gsfc.nasa.gov).

This picture demonstrates that in 2012 the bulk of the SW density flux consisted from the slow SW of nearly $400 \mathrm{~km} \mathrm{~s}^{-1}$, the SW flow distributions of both polarities at the speed of $250-550 \mathrm{~km} \mathrm{~s}^{-1}$ being similar. We assume that such SW density flux may be partly associated with small CHs with different polarities located at low and middle latitudes inside all four coronal field regions (Fig. 3). At the speed greater than $550 \mathrm{~km} \mathrm{~s}^{-1}$, the SW flux of positive polarity exceeded that of the negative polarity above the errors. Using the ICME catalog for 2012 (Richardson \& Cane 2010), we calculated the relative contributions of the transient ICME flows to different velocity components of $\mathrm{SW}$ divided on $50 \mathrm{~km} \mathrm{~s}^{-1}$ bins (Fig. 6, right). It can be seen that in the whole interval from 250 to $750 \mathrm{~km} \mathrm{~s}^{-1}$ the contribution of ICMEs does not exceed $16 \%$. Thus, the enhanced SW flux of positive polarity with $V>550 \mathrm{~km} \mathrm{~s}^{-1}$ is associated with the LCH.

We considered the relationship of the SW density flux with parameters of $\mathrm{CHs}$ and the solar activity separately for the total, slow $\left(V<450 \mathrm{~km} \mathrm{~s}^{-1}\right)$ and fast $\left(V>450 \mathrm{~km} \mathrm{~s}^{-1}\right) \mathrm{SW}$ flux. Figure 7 shows the variations of density fluxes for the slow, fast and total SW averaged for each Carrington rotation in comparison with the averaged sunspots numbers taken from the Solar Influence Data Center in Brussels (SIDC, http://www.sidc.be). The averaged sunspots numbers were calculated as averaged daily numbers of sunspots in each rotation.

The total SW density flux averaged over CR presented in Figure 7 varies between 0.5 and $1.5 \times 10^{8} \mathrm{~cm}^{-2} \mathrm{~s}^{-1}$ with the mean value being around $1 \times 10^{8} \mathrm{~cm}^{-2} \mathrm{~s}^{-1}$. In the beginning of the year (CR 2119) the fast wind constituted about $8 \%$ of the total SW density flux, in the middle of the year (CR 2124-2125) this component increased to $32 \%$ and then in the end of the year (CR 2127-2129) it decreased back to $3 \%$ of the total flux. Averaged over the year, the fast component was about $10 \%$ of the total SW density flux, which is less than the value averaged over longer temporal periods (Wang 2010a). The slow-speed flux constituted from $92 \%$ of the total flux during CR 2119 to $68 \%$ during CR 2125 and again to $97 \%$ during CR 2130. Both components and the total SW density flux in 2012 were considerably lower than the long period averages $\left(2-4 \times 10^{8} \mathrm{~cm}^{-2} \mathrm{~s}^{-1}\right)$ given by Feldman et al. (1978), Goldstein et al. (1996), and Wang (2010a). 

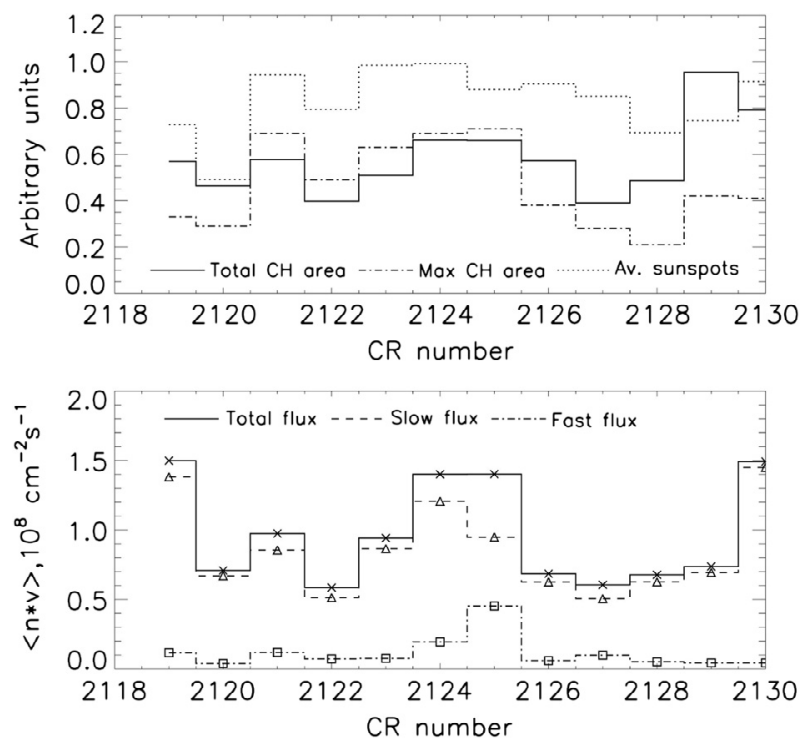

Figure 7. Top panel: variations of the $\mathrm{CH}$ areas during CR 21192130 (January-November 2012) in arbitrary units. Solid line - the total $\mathrm{CH}$ area; dashed-dotted line - the maximum value of the $\mathrm{CH}$ area in the current rotation obtained from the SDO/AIA images at $193 \AA$ A. Dotted line - the daily sunspots numbers averaged over each rotation (in arbitrary units). Bottom panel: variation of the SW density flux during CR 2119-2130. Solid line with crosses - the total SW density flux; dashed line with triangles - the slow SW density flux $\left(V<450 \mathrm{~km} \mathrm{~s}^{-1}\right)$; dashed-dotted line with squares - the fast SW density flux $\left(V>450 \mathrm{~km} \mathrm{~s}^{-1}\right)$.

It is necessary to point out that only the fast SW density flux component evidently correlates with the averaged sunspots number (Table 1), which is associated with the sporadic solar activity, most probably, in the form of CMEs. Fast SW density flux correlates with maximum values of the $\mathrm{CH}$ areas. Slow SW flux correlates better with the total $\mathrm{CH}$ area. The maximum $\mathrm{CH}$ areas are linked to the $\mathrm{LCH}$, which produce SW streams with the velocities more than $450 \mathrm{~km} \mathrm{~s}^{-1}$. Their contribution to the total SW density flux is significant during CR 21212125 (Fig. 7). The total $\mathrm{CH}$ area also includes many small $\mathrm{CHs}$ of different polarities producing the SW streams with the velocities near $450 \mathrm{~km} \mathrm{~s}^{-1}$, which in total can exceed the area of the LCH. So, the slowest and densest SW flows show the best correlation with the total area of CHs.

Figure 8 shows the SW density flux as a function of velocity averaged over the $50 \mathrm{~km} \mathrm{~s}^{-1}$ bins for rotations CR 2119 2130 separately for positive and negative polarity of $B x$ component of the IMF. We can see that the SW density flux with the velocity around $550-650 \mathrm{~km} \mathrm{~s}^{-1}$ with positive polarity shows the notable enhancement during CR 2122-2125 associated with the $\mathrm{LCH}$.

Furthermore, the area of this LCH (Fig. 2b), as well as the solar activity (Fig. 7), declined during CR 2127-2129, which resulted in decrease of the fast component of the SW density flux of positive polarity as well as the total value of SW flux. Thus, structure of the SW density flux and its total value depend on area of the large-scale $\mathrm{CH}$ as well as on level of the solar activity. The distributions in Figure 8 show that the SW density fluxes of different polarities changed significantly from rotation to rotation. The SW density flux distributions are similar for the streams of both polarities only during CR 2122 and 2123 for $V<450 \mathrm{~km} \mathrm{~s}^{-1}$. However, the character of this distribution has changed dramatically starting from
Table 1. Correlation coefficients between components of SW density flux, $\mathrm{CH}$ areas and daily sunspots numbers averaged over Carrington rotations.

\begin{tabular}{lccc}
\hline \hline Flux & Total CH area & Maximum CH area & Av. sunspots \\
\hline Total & 0.44 & 0.43 & 0.08 \\
Fast & 0.10 & 0.61 & 0.38 \\
Slow & 0.46 & 0.27 & -0.04 \\
\hline
\end{tabular}

CR 2126, when the transformation of the quadrupole structure of the coronal magnetic field took place.

\section{Summary}

In conclusion, the obtained results have shown that the multipolar structure of the solar magnetic field and its variations during the polarity reversal process were the main causes of evolution of the coronal sources of SW and, thus, defined the main parameters of space weather in 2012 - configuration of the heliospheric magnetic field and parameters of SW.

The global magnetic field of the corona, large-scale longliving $\mathrm{CH}$, the sector structure of IMF and SW streams near maximum of cycle 24 were studied using the WSO magnetic field measurements and coronal extrapolations as well as the SDO EUV images and the ACE SW data.

The stable quadrupolar structure of the coronal magnetic field was observed during February-August 2012. One of the negative polarity regions contained a long-living transequatorial isolated large $\mathrm{CH}$ ( $\mathrm{LCH})$, being the source of a dominating recurrent high-speed SW stream with a speed of more than $550 \mathrm{~km} \mathrm{~s}^{-1}$. The disappearance of the LCH coincided with the decrease in the quadrupole component strength. The evolution of the $\mathrm{LCH}$ resulted in the variation of the SW properties near the Earth. The distribution of SW velocity versus polarity of the $B x$-component of IMF differed appreciably in time before and after the disappearance of the LCH. We have determined that the equatorial part of the $\mathrm{LCH}$ rotated with a period of 26.9 days, which is slightly less than the rotational period of mid-latitude part of the same LCH (27.3 days). It agrees with the conclusion of Wang et al. (2010b) about rotation of $\mathrm{CHs}$ formed by ARs.

The SW sources during 2012 produced SW fluxes with varying average velocities and densities in the scale of rotations. The mean total SW density flux in 2012 was considerably lower $\left(n V=1 \times 10^{8} \mathrm{~cm}^{-2} \mathrm{~s}^{-1}\right)$ than the typical long-term value of $2-4 \times 10^{8} \mathrm{~cm}^{-2} \mathrm{~s}^{-1}$ (Feldman et al. 1978; Goldstein et al. 1996). According to the assumption of Wang (2010a), such low SW density flux could result from lower coronal heating due to the weaker magnetic field at the coronal base. It confirms the tendency described by McComas et al. (2008), who have pointed out that in the minimum of the current solar cycle the SW density flux measured by Ulysses was lower than in the previous minimum by as much as $\sim 20 \%$. The high-speed SW flux with $V>450 \mathrm{~km} \mathrm{~s}^{-1}$ constituted $32 \%$ as maximum during the existence of the $\mathrm{LCH}$, but only $10 \%$ in the average of the total SW density flux value. The slow-speed component of SW density flux comprised more than $68 \%$ of the total flux.

The structure of the SW density flux and its total value depended on the area of the CHs as well as on the level of solar activity. A significant positive correlation existed between the 
CR2119

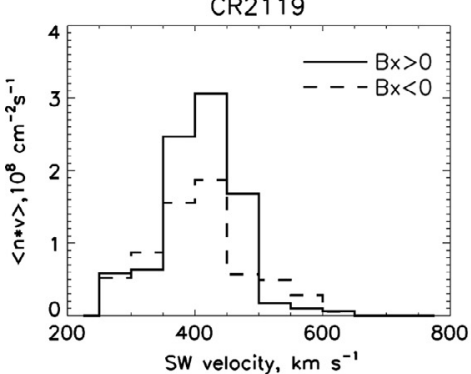

CR2122

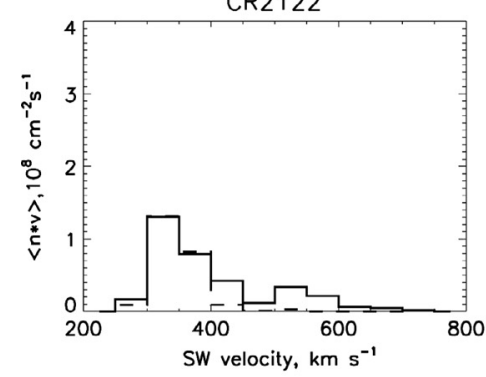

CR2125

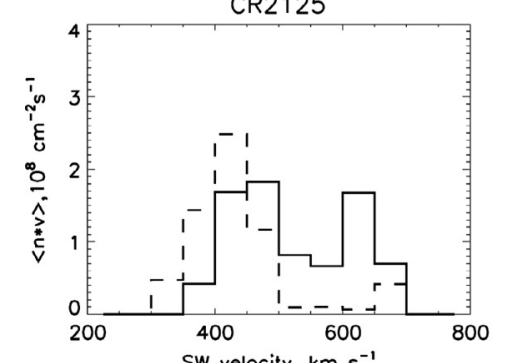

SW velocity, $\mathrm{km} \mathrm{s}^{-1}$

CR2128

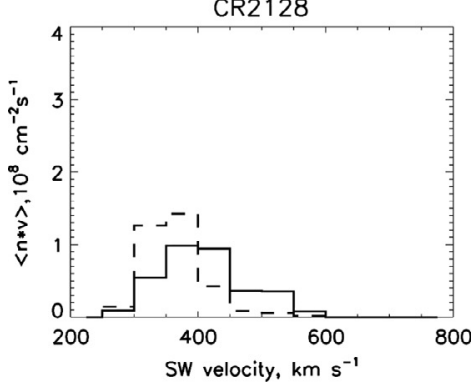

CR2120

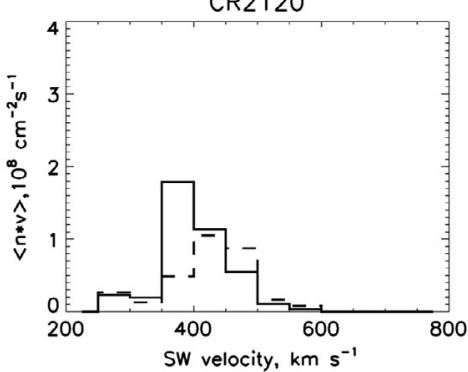

CR2123

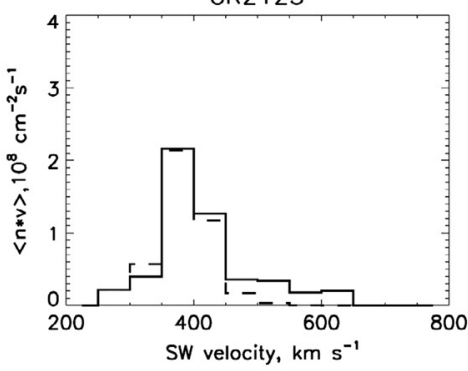

CR2 126

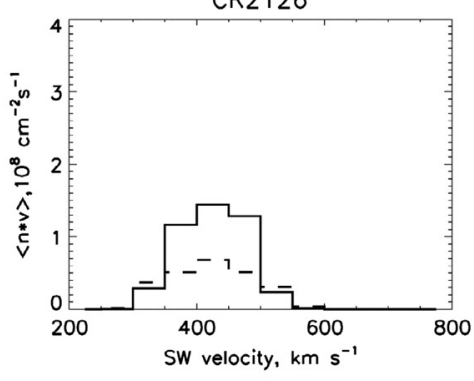

CR2129

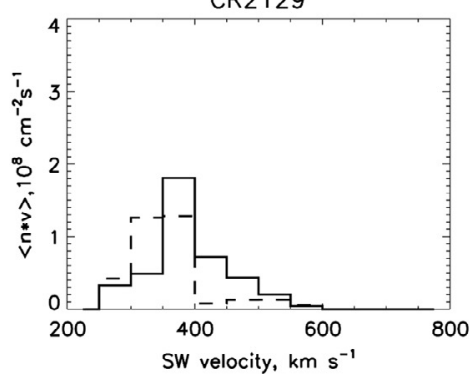

CR2121

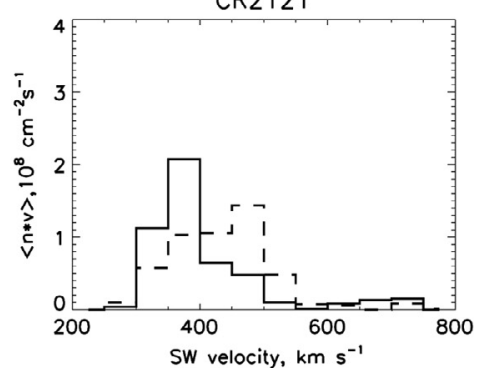

CR2124

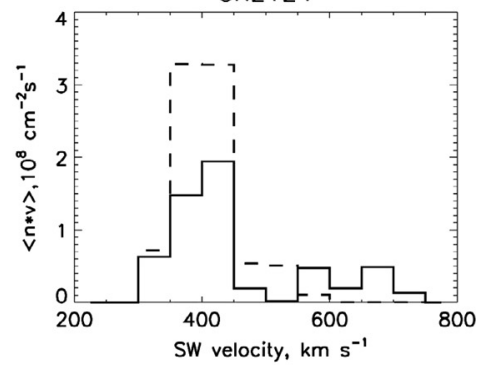

CR2127

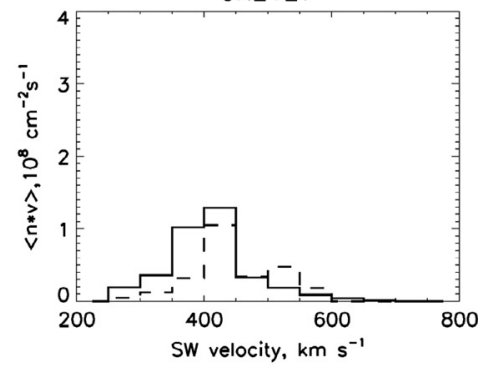

CR2130

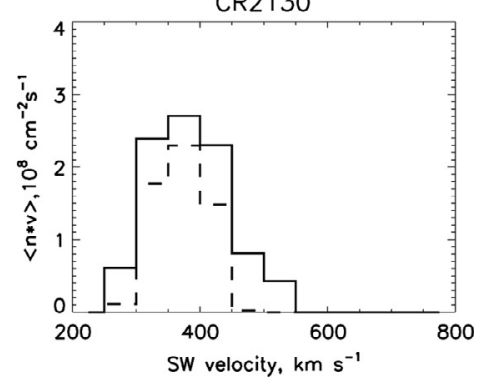

Figure 8. Distribution of the total SW density flux averaged over Carrington rotations versus SW velocity with positive (sold line) and negative (dash line) $B x$-component of the IMF during CR 2119-2130.

high-speed density flux of SW streams and the maximum $\mathrm{CH}$ area determined from the SDO/AIA $193 \AA$ images. Taking the data averaged over rotations, the Pearson correlation coefficient reached more than 0.6 for the high-speed SW streams with the velocity of more than $450 \mathrm{~km} \mathrm{~s}^{-1}$ (Table 1). For slow SW streams with $V<450 \mathrm{~km} \mathrm{~s}^{-1}$ the correlation with the maximum $\mathrm{CH}$ area was less than 0.3. The slow SW flux showed higher correlation $(>0.4)$ with the total $\mathrm{CH}$ area independently of their polarity which suggests that these flows can be associated with many small CHs scattered over the Sun in the regions of different polarity. The relative contributions of the transient ICME flows on the distribution of SW density flux did not exceed $11 \%$ for the slow SW and increased to $16 \%$ for the high-speed SW in 2012.

Acknowledgements. We are thankful to Dr. Yi-Ming Wang and an anonymous Referee for their comments and suggestions. We are grateful to P.H. Scherrer, T. Hoeksema and WSO staff for providing open access to their data and products. We also appreciate the
SIDC-team, World Data Center for the Sunspot Index, Royal Observatory of Belgium, Monthly Report on the International Sunspot Number, online catalogue of the sunspot index. The data from the Solar Dynamics Observatory (SDO) was provided by NASA/SDO and by the AIA, EVE, and HMI science teams. The work was in part supported by the RFBR Grants 13-02-00461 and 14-02-00945a, the Programs P22, P26, and OFN-15 of the Russian Academy of Sciences, the FP-7 eHEROES Project No. 284461 of the European Commission.

\section{References}

Bilenko, I.A., Coronal holes and the solar polar field reversal, $A \& A$, 396, 657-666, DOI: 10.1051/0004-6361:20021412, 2002.

Del Zanna, G., Flows in active region loops observed by Hinode EIS, $A \& A$, 481, L49-L52, DOI: 10.1051/0004-6361:20079087, 2008.

Feldman, W.C., J.R. Asbridge, S.J. Bame, and J.T. Gosling, Longterm variations of selected solar wind properties: $\operatorname{Imp} 6,7$, and 8 results, J. Geophys. Res., 83 (A5), 2177-2189, 1978. 
Goldstein, B.E., M. Neugebauer, J.L. Phillips, S Bame, J.T. Gosling, D. McComas, Y.M. Wang, N.R. Sheeley, and S.T. Suess, ULYSSES plasma parameters: latitudinal, radial, and temporal variations, $A \& A$, 316, 296-303, 1996.

Harra, L.K, T. Sakao, C.H. Mandrini, H. Hara, S. Imada, P.R. Young, L. van Driel-Gesztelyi, and D. Baker, Outflows at the edges of active regions: contribution to solar wind formation? Astrophys. $J$. Lett., 676, L147-L150, DOI: 10.1086/587485, 2008.

Hoeksema, J.T., J.M. Wilcox, and P.H. Scherrer, Structure of the heliospheric current sheet in the early portion of sunspot cycle 21 , J. Geophys. Res., 87 (A12), 10331-10338, 1982.

Hurlburt, N., M. Cheung, C. Schrijver, L. Chang, S. Freeland, et al., Heliophysics event knowledgebase for the Solar Dynamics Observatory (SDO) and beyond, Sol. Phys., 275, 67-78, DOI: 10.1007/s11207-010-9624-2, 2012.

Kojima, M., K. Fujiki, T. Ohmi, M. Tokumaru, and A. Yokobe, Low-speed solar wind from the vicinity of solar active regions, J. Geophys. Res., 104 (A8), 16993-17004, DOI: 10.1029/ 1999JA900177, 1999.

Krista, L., and P. Gallagher, Gallagher Automatic coronal hole detection using local intensity thresholding techniques, Sol. Phys., 256, 87-100, 2009.

Liewer, P.C., J.R. Hall, M.D Jong, D.G. Socker, R.A. Howard, P.C. Crane, P. Reiser, N. Rich, and A. Vourlidas, Determination of three-dimensional structure of coronal streamers and relationship to the solar magnetic field, J. Geophys. Res., 106, 15903-15915, DOI: $10.1029 / 2000 J A 000110,2001$

Liewer, P.C., M. Neugebauer, and T. Zurbuchen, Characteristics of active-region sources of solar wind near solar maximum, Sol. Phys., 223, 209-229, DOI: 10.1007/s11207-004-1105-z, 2004.

MacNeice, P., B. Elliott, and A. Acebal, Validation of community models: 3. Tracing field lines in heliospheric models, Space Weather, 9 (15), DOI: 10.1029/2011SW000665, 2011.

McComas, D.J., S.J. Bame, P. Barker, W.C. Feldman, J.L. Phillips, P. Riley, and J.W. Griffee, Solar Wind Electron Proton Alpha Monitor (SWEPAM) for the advanced composition explorer, Space Sci. Rev., 86, 563-612, DOI: 10.1023/A:1005040232597, 1998.

McComas, D.J., R.W. Ebert, H.A. Elliott, B.E. Goldstein, J.T. Gosling, N.A. Schwadron, and R.M. Skoug, Weaker solar wind from the polar coronal holes and the whole Sun, Geophys. Res. Lett., 35, L18103, DOI: 10.1029/2008GL034896, 2008.

Mogilevsky, E.I., V.N. Obridko, and N.S. Shilova, Large-scale magnetic field structures and coronal holes on the sun, Sol. Phys., 176, 107-121, 1997.

Neugebauer, M., P.C. Liewer, E.J. Smith, R.M. Skoug, and T.H. Zurbuchen, Sources of the solar wind at solar activity maximum, J. Geophys. Res., 107 (A12), 1488, DOI: 10.1029/2001JA000306, 2002.

Nolte, J.T., and E.C. Roelof, Large-scale structure of the interplanetary medium, Sol. Phys., 33, 241-257, DOI: 10.1007/BF00152395, 1973.

Obridko, V.N., B.D. Shelting, I.M. Livshits, and A.B. Asgarov, Contrast of coronal holes and parameters of associated solar wind streams, Sol. Phys., 260, 191-206, DOI: 10.1007/s11207-009-9435-5, 2009.

Obridko, V.N., and B.D. Shelting, Relationship between the parameters of coronal holes and high-speed solar wind streams over an activity cycle, Sol. Phys., 270, 297-310,

DOI: 10.1007/s11207-011-9753-2, 2011.
Owens, M.J., N.U. Crooker, and M. Lockwood, Solar origin of heliospheric magnetic field inversions: Evidence for coronal loop opening within pseudostreamers, J. Geophys. Res., 118, 1868-1879, DOI:10.1002/jgra.50259, 2013.

Sanderson, T.R., T. Appourchaux, J.T. Hoeksema, and K.L. Harvey, Observations of the sun's magnetic field during the recent solar maximum, J. Geophys. Res., 108 (A1), 1035, DOI: 10.1029/2002JA009388, 2003.

Sheeley, N.R., Y.-M. Wang, S.H. Hawley, G.E. Brueckner, K.P. Dere et al., Measurements of flow speeds in the corona between 2 and 30 Rsun, Astrophys. J., 484, 472-478, DOI: 10.1086/304338, 1997.

Shelke, R.N., and M.C. Pande, Differential rotation of coronal holes, Sol. Phys., 95, 193-197, 1985.

Shugay, Yu.S., I.S. Veselovsky, D.B. Seaton, and D. Berghmans, Hierarchical approach to forecasting recurrent solar wind streams, Solar Syst. Res., 45 (6), 546-556, DOI: 10.1134/S0038094611060086, 2011.

Slemzin, V., L. Harra, A. Urnov, S. Kuzin, F. Goryaev, and D. Berghmans, Signatures of slow solar wind streams from active regions in the inner corona, Sol. Phys., 286, 157-184, DOI: 10.1007/s11207-012-0004-y, 2013.

Smith, C.W., M.H. Acuna, L.F. Burlaga, J. L'Heureux, N.F. Ness, and J. Scheifele, The ACE magnetic field experiment, Space Sci. Rev., 86, 613-632, DOI: 10.1023/A:1005092216668, 1998.

Svalgaard, L., and Y. Kamide, Asymmetric solar polar field reversal, Astrophys. J., 763 (23), DOI: 10.1088/0004-637X/763/1/23, 2013.

Richardson, I.G., and H.V. Cane, Near-Earth interplanetary coronal mass ejections during solar cycle 23 (1996-2009): catalog and summary of properties, Sol. Phys., 264, 189-237, DOI: 10.1007/s11207-010-9568-6, 2010.

Veselovsky, I.S., I.G. Persiantsev, and Y.S. Shugai, Forecast of the solar wind velocity and the interplanetary magnetic field radial component polarity at the phase of decay of solar cycle 23 , Geomag. Aeron., 46 (6), 701-707, DOI: $10.1134 / \mathrm{S} 001679320606003 \mathrm{X}, 2006$.

Vrsnak, B., M. Temmer, and A.M. Veronig, Coronal holes and solar wind high-speed streams: I. forecasting the solar wind parameters, Sol. Phys., 240, 315-330, DOI: 10.1007/s11207-007-0285-8, 2007.

Wang, Y.-M., and N.R. Sheeley Jr, Footpoint switching and the evolution of coronal holes, Astrophys. J., 612, 1196-1205, DOI: 10.1086/422711, 2004.

Wang, Y.-M., Coronal holes and open magnetic flux, Space Sci. Rev., 144, 383-399, DOI: 10.1007/s11214-008-9434-0, 2009.

Wang, Y.-M., On the relative constancy of the solar wind mass flux at 1 AU, Astrophys. J. Lett., 715, L121, DOI: $10.1088 / 2041-8205 / 715 / 2 / L 121,2010 a$

Wang, Y.-M., E. Robbrecht, A.P. Rouillard, N.R. SheeleyJr., and A.F.R. Thernisien, Formation and evolution of coronal holes following the emergence of active regions, Astrophys. J., 715, 39, DOI: $10.1088 / 0004-637 X / 715 / 1 / 39,2010 b$

Wang, Y.-M., P.R. Young, and K. Muglach, Evidence for two Separate heliospheric current sheets of cylindrical shape during Mid-2012, Astrophys. J., 780, 103,

DOI: 10.1088/0004-637X/780/1/103, 2014.

Cite this article as: Shugay Y, Slemzin V \& Veselovsky I: Magnetic field sector structure and origins of solar wind streams in 2012. J. Space Weather Space Clim., 2014, 4, A24. 